\title{
Drug Refills
}

National Cancer Institute

\section{Source}

National Cancer Institute. Drug Refills. NCI Thesaurus. Code C127111.

The number of refills permitted for a prescribed medication. 